\title{
BILATERAL VERSUS SINGLE LUNG TRANSPLANTATION FOR CHRONIC OBSTRUCTIVE PULMONARY DISEASE
}

Joseph E. Bavaria, MD

Robert Kotloff, MD

Harold Palevsky, MD

Bruce Rosengard, MD

John R. Roberts, MD

Peter M. Wahl, BA

Nancy Blumenthal, RN

Christine Archer, RN

Larry R. Kaiser, MD
Objective: Traditionally, despite ventilation/perfusion mismatch, single lung transplantation has been the mainstay for end-stage chronic obstructive pulmonary disease. We tested the hypothesis that bilateral sequential lung transplantation has better short- and intermediate-term results than single lung transplantation for chronic obstructive pulmonary disease. Methods: One hundred twenty-six consecutive lung transplants have been performed from November 1991 to March 1996. Seventy-six have been for chronic obstructive pulmonary disease. The diagnosis of this disease includes emphysema $(80.3 \%), \alpha_{1}$-antitrypsin deficiency $(9.2 \%)$, lymphangioleiomyomatosis $(7.9 \%)$, and obliterative bronchiolitis $(2.6 \%)$. Twenty-nine transplants have been bilateral and 47 have been single. Mean age was 55.3 for patients having single lung transplantation and $\mathbf{4 8 . 8}$ for those having bilateral lung transplantation $(p=0.001)$. The distribution of the diagnoses was similar between the two groups. At 6 months, there were 29 survivors of single lung transplantation and 20 survivors of bilateral lung transplantation, with complete data for evaluation. Pulmonary function tests and 6-minute walk tests were evaluated at a mean of 15.4 and 12.8 months after transplantation, respectively. Results: Sixty-day mortality was $\mathbf{2 1 . 3 \%}$ for single lung transplantation versus only $3.45 \%$ for bilateral lung transplantation ( $p=0.03$ ). Additionally, Kaplan-Meier analysis revealed 1and 2-year survivals of $71.1 \%$ and $63.3 \%$ for single lung transplantation versus $90 \%$ and $90 \%$ for bilateral lung transplantation, respectively. Multiple major morbidities were analyzed. Primary graft failure was significantly reduced in the bilateral group $(p=0.049)$. Both 6 -minute walk tests and forced expiratory volume in 1 second were improved from baseline by both single and bilateral lung transplantation $(p=0.001)$. Conclusions: Bilateral lung transplantation improves forced expiratory volume in 1 second and 6-minute walk tests significantly over single lung transplantation $(p<0.0001$ ). Both perioperative mortality and Kaplan-Meier survival (to 3 years) are significantly improved when bilateral rather than single lung transplantation is used for chronic obstructive pulmonary disease in our series $(p<0.05)$. This is probably the result of significantly reduced primary graft failure. (J Thorac Cardiovasc Surg 1997;113:520-8)
From the Departments of Cardiothoracic Surgery and Pulmonary Medicine, University of Pennsylvania Medical Center, Philadelphia, Pa.

Read at the Seventy-sixth Annual Meeting of The American Association for Thoracic Surgery, San Diego, Calif., April 28-May 1, 1996.

Received for publication May 13, 1996; revisions requested July 1, 1996; revisions received Oct. 30, 1996; accepted for publication Nov. 5, 1996.

Address for reprints: Joseph E. Bavaria, MD, Department of Surgery, 4 Silverstein, Hospital of the University of Pennsylvania, 3400 Spruce Street, Philadelphia, PA 19104.

Copyright (C 1997 by Mosby-Year Book, Inc.

$0022-5223 / 97 \$ 5.00+0 \quad \mathbf{1 2 / 6 / 7 9 0 4 3}$
Clinical lung transplantation is indicated for several end-stage pulmonary conditions. They broadly include (1) septic lung disease, (2) pulmonary vascular disease, (3) restrictive lung disease, and (4) chronic obstructive pulmonary disease (COPD). Some indications include disease processes that encompass two or more of these subcategories (i.e., sarcoid). It was only natural that the first clinical lung transplant success for pulmonary fibrosis by Cooper and associates ${ }^{1}$ was followed by renewed attempts to perform lung transplantation for COPD. ${ }^{2}$ The initial successful lung transplanta- 
Table I. Preoperative group demographics

\begin{tabular}{lccl}
\hline & $B L T$ & \multicolumn{1}{c}{$S L T$} & $p$ Value \\
\hline Age & $48.8 \pm 9.7$ & $55.3 \pm 6.6$ & 0.001 \\
Sex (\% male) & $17 / 29(58.6 \%)$ & $13 / 47(27.7 \%)$ & 0.007 \\
$\mathrm{PCO}_{2}$ (mm Hg) & $46.5 \pm 11.9$ & $49.3 \pm 10.5$ & $0.15(\mathrm{NS})$ \\
$\mathrm{PAP}(\mathrm{mm} \mathrm{Hg})$ & $37.9 \pm 11.2$ & $35.8 \pm 7.01$ & $0.19(\mathrm{NS})$ \\
FVC (L) & $2.18 \pm 0.75$ & $1.76 \pm 0.49$ & 0.0097 \\
FEV $_{1}$ (L) & $0.6 \pm 0.17$ & $0.55 \pm 0.18$ & $0.096(\mathrm{NS})$ \\
RV (L) & $5.41 \pm 2.1$ & $5.13 \pm 1.32$ & $0.33(\mathrm{NS})$ \\
6MWT (feet) & $712.7 \pm 238.1$ & $677.1 \pm 290.8$ & $0.26(\mathrm{NS})$ \\
\hline
\end{tabular}

Data are shown as mean \pm the standard deviation. $\mathrm{PCO}_{2}$, Carbon dioxide tension; $P A P$, pulmonary artery pressure; $F V C$, forced vital capacity; $F E V$, volume exhaled in 1 second; $R V$, residual volume; $6 M W T$, 6-minute walk test; $N S$, not significant. Pulmonary function test data were measured at the time of the initial evaluation and listing.

tion operations for COPD included both heart-lung transplantation and double lung transplantation., ${ }^{3,4}$ Two lungs were thought to be necessary to alleviate any severe ventilation/perfusion mismatch or potential hyperinflationary mediastinal complications theoretically possible with single lung transplantation (SLT) ${ }^{5,6}$ In 1989, Mal and associates ${ }^{7}$ reported the first clinical series of isolated SLT for COPD. A number of other reports followed thereafter, including the contribution of the San Antonio group. ${ }^{8-10}$ After these successful reports, there was significant enthusiasm for SLT as the procedure of choice for COPD. ${ }^{11}$ The bilateral sequential lung transplantation procedure (BLT) was then developed and reported in 1990 with excellent results. ${ }^{12,13}$ Since that time, over the past 3 to 4 years, a debate has been in progress regarding the question: Is BLT or SLT the optimal procedure for patients with endstage COPD? ? $^{3,14}$ This is not simply a medical debate, because there are ethical and donor organ issues as well. ${ }^{11}$

This report analyzes, in a retrospective fashion, the results in our program comparing the outcomes of SLT and BLT for end-stage obstructive lung disease. Our decision algorithm for choosing BLT versus SLT for COPD has evolved since our initial lung transplant procedure for COPD in December 1991. The intent of this analysis is to compare our consecutive series of BLTs with SLTs over the past 4.25 years.

\section{Patients and methods}

Patients. Between November 1991 and March 1996, 126 total lung transplants were performed by the Hospital of the University of Pennsylvania Lung Transplant Program in 123 patients. Seventy-six patients were operated on with a diagnosis of COPD. The diagnosis of COPD is

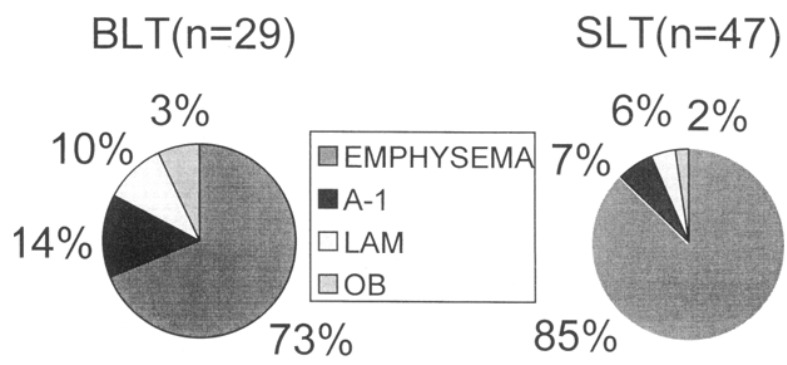

Fig. 1. Comparison of distribution of diagnoses in the two transplant recipient groups. There was no difference between groups $(p=0.09) . A-1, \alpha_{1}$-antitrypsin deficiency emphysema; $L A M$, lymphangioleiomymatosis; $O B$, obliterative bronchiolitis.

defined to include generalized emphysema, $\alpha_{1}$-antitrypsin deficiency, lymphangioleiomyomatosis, and obliterative bronchiolitis. The vast majority of patients $(>90 \%)$ have emphysema or $\alpha_{1}$-antitrypsin deficiency. This series is a retrospective analysis of these 76 consecutive patients with COPD. One patient, who committed suicide at home with excellent pulmonary function after SLT, was excluded from the follow-up analysis.

The first SLT for COPD was performed on December 16, 1991 (transplant No. 2 in our program), and the first BLT for COPD was on July 13, 1993 (transplant No. 20 in our program).

Methods. Pulmonary function, exercise ability, and hemodynamics were evaluated in all patients according to a standardized pretransplantation and posttransplantation evaluation. This report examines the changes in forced expiratory volume in 1 second, forced vital capacity, residual volume, six-minute walk test, maximum oxygen uptake, blood gas determination including carbon dioxide tension, and pulmonary artery pressure (measured in millimeters of mercury). All preoperative values were measured at the time of initial evaluation and listing. The mean time to transplantation was more than a year from the time of listing. Surviving patients are evaluated at 3-month intervals. Mean follow-up time for the entire series of surviving patients was 14.6 months.

Study design. Seventy-six patients were evaluated in this study, $61.8 \%$ of all patients who received lung transplantation from November 1991 to March 1996. The frequency of COPD has remained constant over the course of the entire program. Before September 1, 1994 $61.5 \%$ of the recipients had COPD (40/65) versus $60 \%$ from September 1, 1994, to March 21, 1996 (36/60). This was somewhat surprising inasmuch as we anticipated a reduction in the percentage of COPD diagnoses in the latter portion of our experience owing to the increased use of volume reduction as a bridge and alternative to lung transplantation. Most patients (but not all) who received a volume reduction as a bridge were removed from the active lung transplant list. Of the 76 recipients with COPD, the BLT group $(n=29,38.2 \%)$ received bilateral sequential lung transplants and SLT group $(n=47$, $61.8 \%)$ received single lung transplants. The distribution 


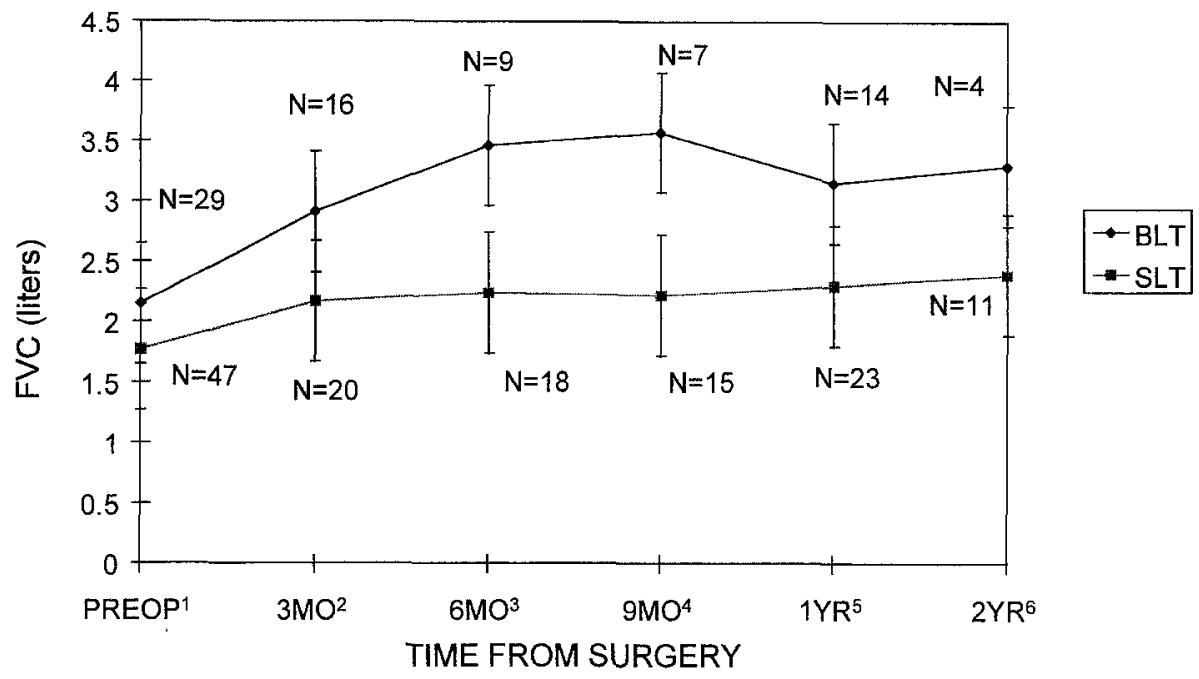

Fig. 2. Forced vital capacity $(F V C)$ before and after SLT and BLT. Standard error bars shown. Decreasing $N$ numbers reflect number of patients tested at that time. ${ }^{1} p=0.0097 ;{ }^{2} p=0.008 ;{ }^{3} p=0.006 ;{ }^{4} p=0.003$; ${ }^{5} p=0.002 ;{ }^{6} p=0.024$.

Table II. Morbidity and major complications

\begin{tabular}{llll}
\hline & \multicolumn{1}{c}{ BLT } & SLT & $p$ Value \\
\hline Length of intubation (days) & $2.43 \pm 1.75$ & $8.13 \pm 17.6$ & 0.016 \\
Length of stay (days) & $35.6 \pm 40.4$ & $30.7 \pm 39.5$ & 0.31 (NS) \\
Obliterative bronchiolitis & $2 / 28(7.1 \%)$ & $5 / 37(13.5 \%)$ & 0.4 (NS) \\
Primary graft dysfunction & $1 / 29(3.45 \%)$ & $9 / 47(19.2 \%)$ & 0.049 \\
Cerebrovascular accident & $5 / 29(17.2 \%)$ & $2 / 47(4.3 \%)$ & 0.057 (NS) \\
Tracheostomy & $4 / 29(13.8 \%)$ & $9 / 47(19.2 \%)$ & 0.5 (NS) \\
Anastomosis & $3 / 58(5.2 \%)$ & $1 / 47(2.13 \%)$ & 0.41 (NS) \\
GI/OR & $1 / 29(3.45 \%)$ & $7 / 47(14.9 \%)$ & 0.1 (NS)
\end{tabular}

The obliterative bronchiolitis rate was calculated only for 60-day survivors. Anastomosis indicates the total number of anastomoses at risk necessitating operation, stenting, dilation, or laser therapy; $G I / O R$, gastrointestinal complications necessitating laparotomy or therapeutic endoscopy or a gastrointestinal hemorrhage of greater than 6 units that was treated conservatively; NS, not significant.

of patients within the various categories of COPD is shown in Fig. 1.

Immunosuppression. Our immunosuppression protocols have been based on a three-drug regimen. for the duration of our transplant program. This regimen has included cyclosporine dosed to a level in the first year of approximately 250 to $350 \mathrm{ng} / \mathrm{ml}$ (after the first year it is reduced to 200 to $300 \mathrm{ng} / \mathrm{ml}$ ), azathioprine $2 \mathrm{mg} / \mathrm{kg}$ daily, and prednisone, starting at $0.5 \mathrm{mg} / \mathrm{kg}$ daily and tapering to $0.15 \mathrm{mg} / \mathrm{kg}$ per day by 6 months after transplant. At the very beginning of our program, we used routine induction antithymocyte gamma-globulin therapy instead of prednisone. Prednisone was then begun on the sixth postoperative day. However, we changed to routine perioperative steroid immunosuppression very early in our experience (July 1992). Since transplant No. 60, we have added a "mini-induction" four-drug protocol for the first 72 hours, because we have noted variable cyclosporine levels in the early postoperative period. This "miniinduction" technique added perioperative antithymocyte gamma-globulin to our standard azathioprine (Imuran), cyclosporine, and methylprednisolone sodium succinate (Solu-Medrol) until therapeutic cyclosporine levels were achieved.

Algorithm regarding decision to perform SLT versus BLT. The algorithm regarding the decision process of whether to perform an SLT versus a BLT evolved during the period of this study. Early in our program the indication for BLT for patients with COPD was limited to young patients (aged $<45$ years) with the diagnosis of $\alpha_{1}$-antitrypsin deficiency. By July 1993, the general indications for BLT had evolved to include (1) all patients with the diagnosis of $\alpha_{1}$-antitrypsin deficiency and (2) all patients younger than 50 years of age. Presently (March 1996), we also perform BLT on all patients with lymphangioleiomyomatosis in addition to $\alpha_{1}$-antitrypsin deficiency and strongly consider BLT in all patients between 50 and 59 years of age.

Operative and anesthetic technique. The overwhelming majority of patients having SLT were operated on via 


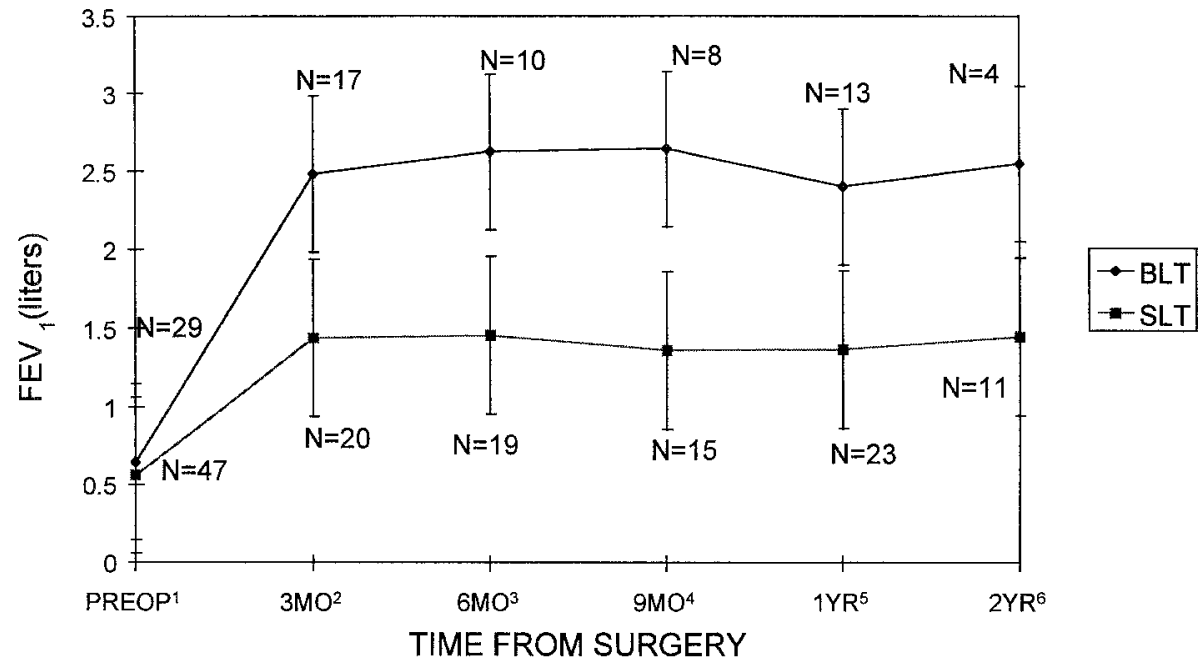

Fig. 3. Forced expiratory volume in 1 second $\left(F E V_{1}\right)$ before and after SLT and BLT. Standard error bars shown. ${ }^{1} p=0.096$ (not significant) ${ }^{2} p=2.38 \times 10^{-4} ;{ }^{3} p=3.09 \times 10^{-5} ;{ }^{4} p=2.13 \times 10^{-5} ;{ }^{5} p=9.34 \times 10^{-5}$; ${ }^{6} p=0.02$.

a standard posterolateral thoracotomy. Cardiopulmonary bypass was not necessary for any of the SLT procedures. Recently we have favored use of a smaller anterolateral muscle-sparing thoracotomy for SLT $(n=$ 5). Since July 1, 1995, patients have received an anesthetic technique that allows for extubation on the operating room table if possible $(n=7)$ for SLT recipients. Intravenous prostaglandin $\mathrm{E}$ is begun during the operation in all patients.

BLT has been performed via an anterior bilateral thoracosternotomy approach. ${ }^{13}$ Only one patient $(3.3 \%$ ) required cardiopulmonary bypass during BLT for COPD. No BLT recipient has been extubated on the operating room table. Both BLT and SLT recipients are subjected to a postproceduse therapeutic bronchoscopy in the operating room and just before extubation.

Statistical analyses. Student's $t$ tests were used when comparing continuous numerical strings such as age, length of in-hospital stay, length of intubation, carbon dioxide tension, oxygen tension, maximum oxygen uptake, pulmonary function, and 6-minute walk test data between groups. $\chi^{2}$ Analysis was used to determine statistical significance of single-event, noncontinuous indices such as morbidity and mortality rates. Kaplan-Meier survival estimates required the use of the log rank (Mantel-Cox) method of $\chi^{2}$ analysis to determine significance, comparing BLT versus SLT survivals. Statistical significance was defined as a $p$ value less than 0.05 .

\section{Results}

Definitions. The entire experience of this lung transplant program was reviewed. One hundred twenty-six transplant procedures were performed in 123 recipients. Seventy-six recipients $(61.8 \%)$ had COPD. In our cohort of 76 recipients with
COPD, 29 (38.2\%) received BLTs (BLT group) and $47(61.8 \%)$ received SLTs (SLT group). The broad definition of COPD considered for this study included emphysema, $\alpha_{1}$-antitrypsin deficiency emphysema, lymphangioleiomyomatosis, and obliterative bronchiolitis. The distribution of diagnoses in the SLT and BLT groups is shown in Fig. 1.

Preoperative clinical characteristics. Clinical indices were compared between the two groups (BLT versus SLT) and are shown in Table I. Mean age was lower in the BLT group and sex distribution was significantly different between the groups. Preoperative functional status and comorbidity were evaluated and compared between BLT and SLT. Table I demonstrates that the SLT cohort was functionally different only with respect to preoperative forced vital capacity. The remainder of the pulmonary function test data show no significant difference between groups. Fig. 1, which shows the distribution of the indications for transplantation in the two groups, demonstrates no difference between BLT and SLT with respect to the underlying diagnosis ( $p=0.09$, not significant) .

Morbidity. Mean length of intubation (BLT $2.43 \pm 1.75$ days versus SLT $8.13 \pm 17.6$ days; $p=$ 0.016 ) was significantly lower in the BLT group, despite the fact that seven recent SLT recipients were extubated on the operating room table. Mean length of in-hospital stay (BLT $35.6 \pm 40.4$ days 


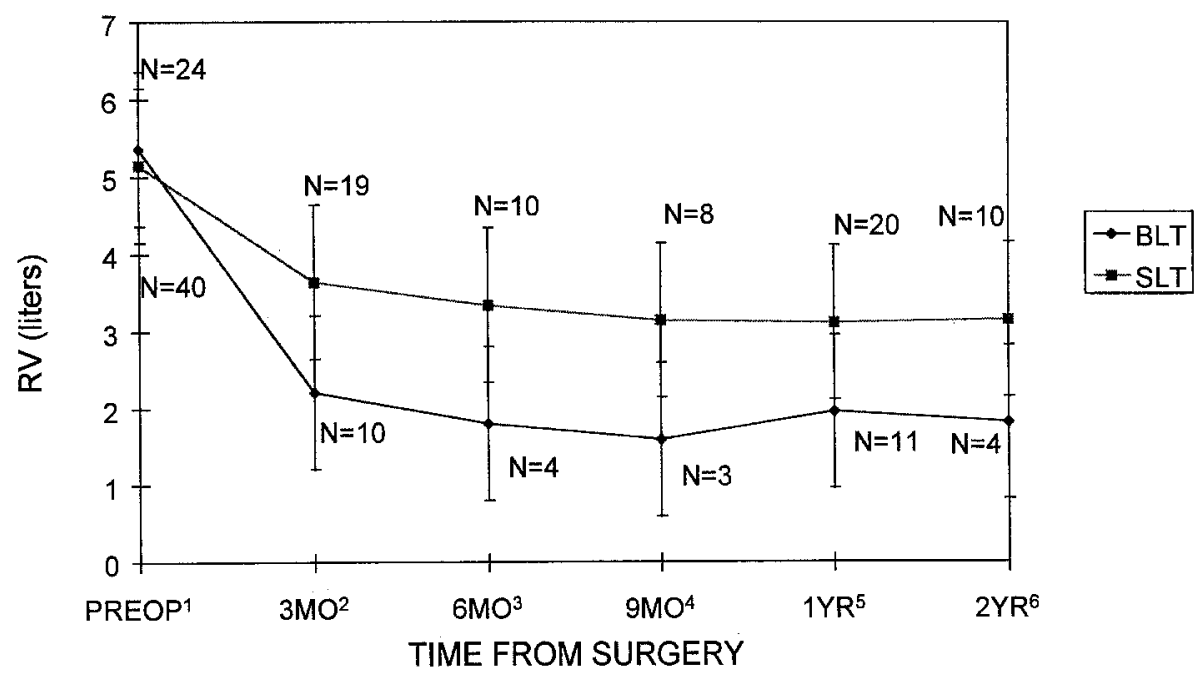

Fig. 4. Residual volume $(R V)$ before and after SLT and BLT. Standard error bars shown. ${ }^{1} p=0.33$ (not significant); ${ }^{2} p=3.12 \times 10^{-6} ;{ }^{3} p=3.83 \times 10^{-4} ;{ }^{4} p=2.13 \times 10^{-5} ;{ }^{5} p=2.06 \times 10^{-6} ;{ }^{6} p=0.002$.

versus SLT $30.7 \pm 39.5$ days; $p=0.31$, not significant) did not differ between the two groups. The incidence of major complications was examined and is shown in Table II. No significant difference was detected between the groups except in the case of primary graft failure, which was significantly lower in the BLT group $(p=0.049)$. Primary graft failure was defined as a form of acute lung injury characterized by the presence of widespread pulmonary infiltrates within lung allograft(s) and associated hypoxemia and ventilatory dependence extending beyond the initial 5 postoperative days. The diagnosis rests on exclusion of other causes of acute lung injury, including pneumonia, aspiration, volumetric overload, hyperacute rejection, massive blood product transfusion, or pulmonary venous outflow obstruction. ${ }^{15}$

There was an increased risk of cerebrovascular accident in the BLT group $(p=0.057)$. Cerebrovascular accident/stroke was defined as any postoperative cerebrovascular accident, including those that resolved without permanent residual deficit. Major gastrointestinal complications were defined as any complication necessitating a laparotomy or therapeutic endoscopic procedure or a gastrointestinal hemorrhage of more than 6 units treated conservatively. Rates of obliterative bronchiolitis, tracheostomy, and bronchial anastomotic complications were no different between procedures. The diagnosis of obliterative bronchiolitis was established on the basis of pulmonary function criteria as recently outlined by Cooper and associates ${ }^{15}$ as a decrement in forced expiratory volume in 1 second of more than $20 \%$ of posttransplantation baseline. ${ }^{15-17}$ In one case in our series, the presence of obliterative bronchiolitis was confirmed histologically by postmortem investigation of the lung. Additionally, phrenic nerve injury was studied and did not differ between groups.

Functional outcome. Paired pulmonary function test data within the two groups were compared after transplantation. Twenty of the $29 \mathrm{BLT}$ recipients and 29 of the 47 SLT recipients had paired data with mean follow-up times of 12.75 and 15.38 months, respectively. Figs. 2 to 5, which show posttransplantation functional and exercise outcomes (forced vital capacity, forced expiratory volume in 1 second, residual volume, and 6-minute walk test distance), demonstrate that improvement in these values was considerably higher for the BLT group (statistical significance shown in legend). Mean maximum oxygen uptake after transplantation (BLT $65.2 \pm 16.6$ $\mathrm{ml} / \mathrm{min}$ versus SLT $61.7 \pm 15.8 \mathrm{ml} / \mathrm{min}$ expressed as a percent of the predicted value; $p=0.29$, not significant) did not differ between the two groups.

Mortality. The 60-day perioperative mortality rate (BLT $1 / 29=3.45 \%$ versus SLT $10 / 47=21.3 \%$; $p=0.03$ ) was lower in the BLT group. Cumulative survivals by Kaplan-Meier analysis, comparing the BLT versus SLT survival curves, were significantly different favoring BLT ( $p=0.047$ ) (Fig. 6).

Of the three patients who died in the BLT group, one died of primary graft failure. The other two died of myocardial infarction and Guillain-Barré syn- 


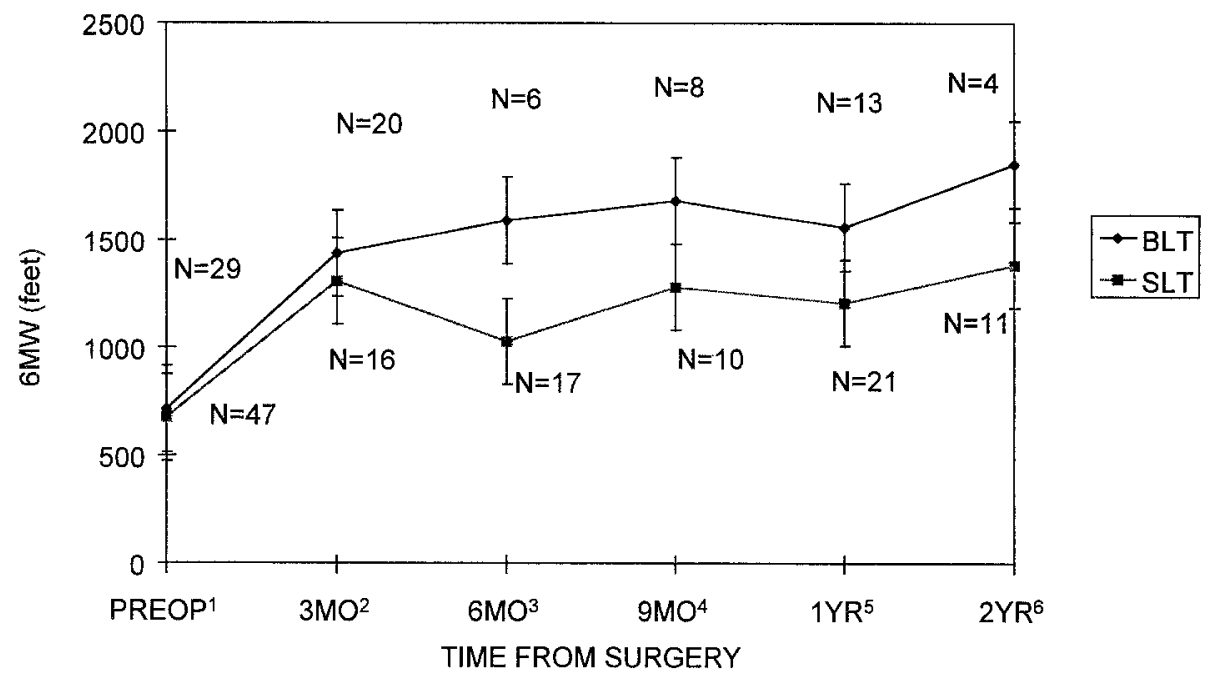

Fig. 5. Comparison of 6-minute walk $(6 M W)$ distance before and after SLT and BLT. Standard error bars shown. ${ }^{1} p=0.26$ (not significant) $;{ }^{2} p=0.16 ;{ }^{3} p=4.43 \times 10^{-4} ;{ }^{4} p=0.06$ (not significant) $;{ }^{5} p=0.015 ;{ }^{6} p=$ 0.022 .

drome at 2 weeks and 10 months, respectively, both with perfectly normal pulmonary function. Conversely, the death rate for SLT recipients includes the earliest initial institutional experience and was significantly affected by the occurrence of primary graft failure. Our initial nine COPD transplants were all SLTs. The perioperative mortality rate was $33 \%(3 / 9)$ and the crude 1-year survival only $44.4 \%$ (4/9). More recent 60 -day mortality is $18.4 \%$ (7/38) if we exclude the first nine patients. These seven patients died of primary graft failure and adult respiratory distress syndrome $(n=4)$, sepsis $(n=$ 2 ), and cytomegalovirus pneumonitis $(n=1)$. Additionally, this $18.4 \%$ perioperative mortality rate can be compared more directly with the BLT group, as these two groups are concurrent, the transplantations having been done during the same time period (July 1993 to April 1996).

Lymphangioleiomyomatosis was present in three patients in both the BLT and SLT groups. Two of the three patients with lymphangioleiomyomatosis in the SLT group died in the perioperative period. One still survives 4 years after transplantation. All three patients with lymphangioleiomyomatosis receiving BLT are survivors. Two patients required transplantation because of obliterative bronchiolitis. Both were perioperative survivors. The SLT recipient died of recurrent obliterative bronchiolitis 15 months after transplantation. The BLT recipient is alive at 2 years.

\section{Discussion}

The history regarding which lung transplant procedure is optimal for patients with end-stage COPD has been one of uncertainty. ${ }^{11,18,19}$ There have been theoretical concerns regarding SLT for COPD resulting from contralateral lung hyperexpansion and severe ventilation/perfusion mismatch since the early 1970s. This theoretical concern was corroborated by multiple reviews and analyses of the lung transplant experience at that time. ${ }^{5,6}$ Initially, after the success of heart-lung transplantation and, subsequently, SLT for pulmonary fibrosis, lung transplantation for COPD was limited to either heartlung transplantation or double lung transplantation, thereby obviating the theoretical concerns of SLT for COPD. The initial reports from France in $1989^{7}$ and subsequent reports from the United States 8,10 showed very successful clinical series of SLT for emphysema. At this time the conceptual framework regarding SLT as a viable transplant procedure for COPD was revisited. ${ }^{9}$ SLT for emphysema was practiced widely, because it was noted that most patients with end-stage COPD from emphysema were between the ages of 50 and 65 years. However, patients considered for double lung transplantation or heart-lung transplantation were younger than 50 years. Debate regarding the optimal lung transplant procedure for COPD resurfaced again after the original development and description of the BLT operation. ${ }^{12}$ This procedure greatly simplified the two- 


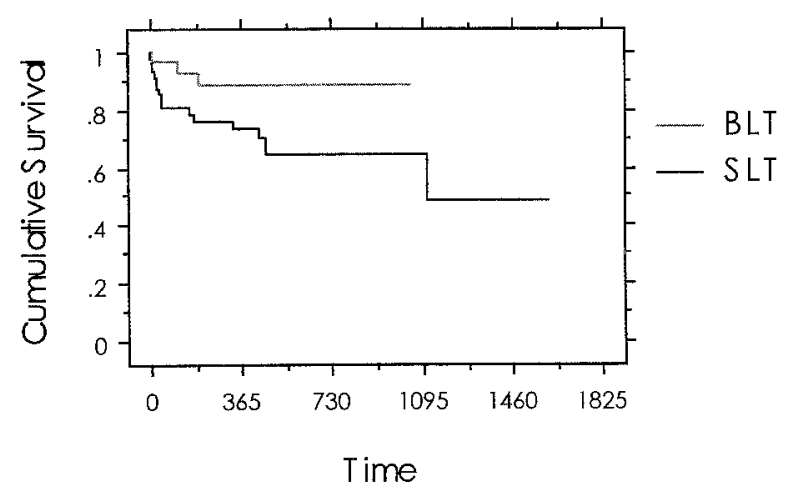

Fig. 6. Kaplan-Meier cumulative survivorship rate curve.

lung transplant procedure, and in most cases it did not necessitate cardiopulmonary bypass and could safely be offered to patients up to 60 years of age. ${ }^{13}$ It was at this point in the evolution of lung transplantation that the competing considerations (improved functional outcome versus donor organ availability) complicated the decision-making process regarding the most appropriate and proper transplant procedure for end-stage COPD.

This report represents an analysis of our consecutive series of lung transplantations for COPD. The dilemma regarding which transplant procedure is optimal for patients with COPD is not resolved. Multiple groups have shown that many pulmonary functional parameters are improved in a statistically significant fashion with BLT over SLT. ${ }^{14,18,20}$ These results include statistically and significantly improved forced expiratory volume in 1 second, forced vital capacity, and residual volume. Our data corroborate these previous reports. Additionally, our data support the concept that not only is standard pulmonary function test data improved with BLT versus SLT, but that exercise tolerance (measured by 6-minute walk tests) is significantly improved. Maximum oxygen uptake is slightly, but not significantly, higher in the BLT group.

When we examine perioperative mortality and morbidity, our data show a statistically significant increase in Kaplan-Meier survival out to 3 and 4 years $(p<0.05)$, as well as a statistically significant decrease in perioperative ( 60 day) mortality with the BLT procedure $(p=0.03)$. We realize that our $21.3 \%$ perioperative SLT mortality rate is higher than expected. We had an early "learning curve," with $33 \%$ of our initial nine patients dying of primary graft failure in the perioperative period. Interestingly, even after eliminating our initial expe- rience and comparing a concurrent set of patients receiving the two procedures, we still had substantially increased perioperative mortality (18.4\%) and primary graft failure $(16.2 \%)$ in the SLT group. Regarding late mortality, we have no BLT deaths after 10 months. Unfortunately, there is a steady death rate after 1 year in the SLT group.

Most major morbidities, as well as length of stay and total intubation time, were analyzed. The BLT group had significantly shorter periods of intubation and a significantly reduced incidence of primary graft dysfunction. The only perioperative morbidity that was increased in the BLT group was stroke, although the increase was not significant $(p=$ $0.057)$. The BLT procedure would theoretically have twice the deairing complications and a left atrial suture line twice the length of that needed for SLT. We have now implemented a policy of preoperative and intraoperative heparin administration with intraoperative transesophageal echocardiography and postoperative subcutaneous heparin and aspirin. Other major morbidities were similar between the two procedures, including phrenic nerve injury, tracheostomy, and obliterative bronchiolitis. Our bronchial anastomotic complication rate has been very low in this entire group of transplant recipients $(3.8 \%)$.

A very important issue regarding the decisionmaking process for patients considered for lung transplantation with COPD is organ availability. It is sometimes difficult to advocate the SLT procedure when our institutional data strongly support the use of BLT for COPD. On the other hand, SLT no doubt will allow more patients to undergo transplantation.

Others have compared the BLT procedure with the SLT procedure. As in our report, the Washington University Lung Transplant Program showed a very similar patient profile between the SLT and BLT groups at evaluation, except for mean age. As in our series, the BLT group was approximately 6 years younger than the SLT group. Their 1-year mortality rate of $10.2 \%$ for SLT in COPD compares favorably with our $28.3 \%$ rate. On the other hand, their $16 \% 1$-year mortality rate for BLT is greater than our $10.3 \%$ rate. $^{18,19}$

In summary, the ideal decision algorithm for determining whether BLT is preferable to SLT is not available. We believe that the BLT procedure confers a definite advantage for pulmonary function. We have also demonstrated a statistically significant increase in exercise tolerance as measured by the 
6-minute walk test and a slightly increased maximum oxygen uptake in the BLT recipients. We have also noted slowly increasing residual volumes in SLT patients with chest $x$-ray evidence of a progressively expanding native lung. There has been a trend toward a decreased overall major complication rate with BLT, especially primary graft failure, although the cerebrovascular accidents are more prevalent. More long-term follow-up at the 3- to 5 -year period will be necessary to make a final determination of whether BLT is better than SLT for end-stage lung disease. However, it is interesting that in our data the BLT group has zero late mortality after 10 months whereas the SLT group has continued mortality after 1 year. Is this a sign that the BLT group tolerates obliterative bronchiolitis, chronic rejection, and cytomegalovirus better than the SLT group? This question needs more analysis. ${ }^{19}$

Presently in our program, we preferentially offer the BLT procedure to all patients with $\alpha_{1}$-antitrypsin deficiency. Additionally, we offer the BLT procedure to all patients with lymphangioleiomyomatosis, and we strongly consider the BLT option for patients younger than 55 years. We even consider BLT selectively for patients between the ages of 55 and 60 , although the three deaths $(10.3 \%)$ in our BLT group occurred in patients who were 60,59 , and 57 years of age. In addition, one of the more severe, nonlethal strokes occurred in a BLT recipient who was 62 years of age. Finally, two 59-year-old BLT recipients, who have since done well, required three major laparotomies for life-threatening gastrointestinal complications. We therefore continue to believe that the simple and very straightforward SLT procedure is optimum in most patients over the age of 55 years. Indeed, recently we have been using muscle-sparing small anterior thoracotomy incisions and intraoperative extubation for most SLT procedures.

We believe that this information supports the recent transplant trend toward increased use of the BLT procedure for COPD. The short- and intermediate-term superiority of BLT over SLT most probably results from an initial reduction in primary graft failure and longer-lasting improvement in pulmonary function.

\section{REFERENCES}

1. Cooper JD, Ginsberg RJ, Goldberg M, The Toronto Lung Transplant Group. Unilateral transplantation for pulmonary fibrosis. N Engl J Med 1986;314:1140-5.
2. Cooper JD, Patterson GA, Grossman R, Maurer J. Doublelung transplant for advanced chronic obstructive lung disease. Am Rev Respir Dis 1989;139:303-7.

3. Patterson GA, Maurer JR, Williams TJ, Cardoso PG, Scavuzzo M, Todd TR. Comparison of outcomes of double and single lung transplantation for obstructive lung disease. J Thorac Cardiovase Surg 1991;101:623-31.

4. Patterson GA. Lung transplantation for chronic obstructive pulmonary disease. Clin Chest Med 1990;11:547-54.

5. Wildevuur CRH, Benfield JR. A review of 23 human lung transplantations by 20 surgeons. Ann Thorac Surg 1970;9: 489-515.

6. Veith FJ, Koerner SK. Problems in the management of human lung transplant patients. Vasc Surg 1974;8:273-82.

7. Mal H, Andreassian B, Pamela F, et al. Unilateral lung transplantation in end stage pulmonary emphysema. Am Rev Respir Dis 1989;140:797-802.

8. Calhoun JH, Grove FL, Gibbons WJ, et al. Single lung transplantation: alternative indications and technique. J Thorac Cardiovasc Surg 1991;101:816-25.

9. Trulock EP, Egan TM, Kouchoukos NT, et al. Single lung transplantation for severe chronic obstructive pulmonary disease. Chest 1989;96:738-42.

10. Marinelli WA, Hertz MI, Shumway SJ, et al. Single lung transplantation for severe emphysema. J Heart Lung Transplant 1992;11:577-83.

11. Low DE, Trulock EP, Kaiser LR, et al. Morbidity, mortality, and early results of single versus bilateral lung transplantation for emphysema. J Thorac Cardiovase Surg 1992;103: 1119-26.

12. Pasque MK, Cooper JD, Kaiser LR, Haydock DA, Triantafilou A, Trulock EP. Improved technique for bilateral lung transplantation: rationale and initial clinical experience. Ann Thorac Surg 1990;49:785-91.

13. Kaiser LR, Pasque MK, Trulock EP, et al. Bilateral sequential lung transplantation: the procedure of choice for doublelung replacement. Ann Thorac Surg 1991;52:438-46.

14. Haydock DA, Trulock EP, Kaiser LR, et al. Lung transplantation: analysis of thirty-six consecutive procedures performed over a twelve month period. J Thorac Cardiovasc Surg 1992;103:329-40.

15. Cooper ID, Billingham M, Egan T, et al. A working formulation for the standardization of nomenclature and for clinical staging of chronic dysfunction in lung allografts. J Heart Lung Transplant 1993;12:713-6.

16. Reichenspurner H, Girgis RE, Robbins RC, et al. Obliterative bronchiolitis after lung and heart-lung transplantation. Ann Thorac Surg 1995;60:1845-53.

17. Sundaresan S, Trulock EP, Mohanakumar T, et al. Prevalence and outcome of bronchiolitis obliterans syndrome after lung transplantation. Ann Thorac Surg 1995;60:1341-7.

18. Gaissert HA, Trulock EP, Cooper JD, Sundaresan RS, Patterson GA. Comparison of early functional results after volume reduction or lung transplantation for chronic obstructive pulmonary disease. J Thorac Cardiovase Surg 1996;111: 296-307.

19. Sundaresan S, Shiraishi X, Manley J, et al. Single or bilateral lung transplantation for emphysema? 1996;112:1485-95.

20. Dromer C, Velly JF, Jougan J, Martigne C, Baudet E, Couraud L. Long-term functional results after bilateral lung transplantation. Ann Thorac Surg 1993;56:68-73. 


\section{Discussion}

Dr. Thomas J. Kirby (Cleveland, Ohio). Would you please clarify your exact rationale for undertaking BLT in the setting of emphysema? BLT is known in the transplant community to give better results than SLT in terms of pulmonary function tests and exercise tolerance. In the abstract you used the word "hypothesis" when comparing the superiority of BLT over SLT. There is nothing hypothetical about the improved functional results of BLT compared with SLT. There may be some controversy in terms of mortality, and that leads to my next question.

The significant mortality that you report in your SLT group is not the experience of others; in fact, at most centers SLT for emphysema carries the lowest mortality. You reported a 60-day mortality of $22.5 \%$ for SLT compared with a $4.2 \% 60$-day mortality for BLT. This is a very unusual experience and should not be used to justify or support the use of BLT in emphysema. In our experience with 36 SLTs carried out for emphysema, we had a 60 -day mortality of $4.7 \%$ and a 1-year survival of $85 \%$. Could you elaborate the reasons for your increased mortality in the SLT group?

Last, and I think most important, donor shortage remains a critical problem in the United States for all major organ transplants and in particular for lung transplantation. The number of patients on waiting lists is rapidly dwarfing the number of lung transplants performed. The waiting list mortality for lung transplantation around the United States varies between $15 \%$ to $20 \%$ per year, which is not an insignificant figure.

Dr. Bavaria, do you think that your improved functional results with BLT justify using two lungs when in most surgeons' hands the two lungs would have been used for two separate recipients?

Dr. Bavaria. Thank you, Dr. Kirby. Those are all very cogent points.

The first question was, why use a BLT. In our experience, we have noticed a decreased incidence of primary graft dysfunction. Increased primary graft dysfunction has led to both increased morbidity and mortality in our SLT group. Only one of 30 patients with BLT had primary graft dysfunction. So I believe it is not just the improved functional parameters that have led us toward bilateral transplantation in this group, but both reduced early graft dysfunction and better long-term results on pulmonary function tests and exercise improvement.

The issue of morbidity and mortality in our experience is a critical one and is actually one of the limitations of this study. We did have a learning curve or an increased mortality rate early on in the experience, but, even so, the BLT group did better than the SLT group. I do not have any other explanation for that except that primary graft dysfunction is the single biggest problem with SLT in our experience, and we simply do not have that problem in our BLT cohort.

Third, I think the answer to the donor availability question is to expand the donor pool. Many of our BLT patients received lungs that we would not have used for an SLT. I resist performing an SLT if the donor has problems and a decreased oxygen tension, say, below $400 \mathrm{~mm} \mathrm{Hg}$, whereas I will use a BLT lung block with an oxygen tension of $250 \mathrm{~mm} \mathrm{Hg}$ because of the increased margin. I think the use of "marginal BLT blocks" may actually increase our donor pool.

Dr. G. Alexander Patterson (St. Louis, Mo.). I was struck by the prevalence of cerebrovascular accidents in your series, five cases in the BLT group. Would you comment on the technique you used to deair the allograft at the time of the procedure? We are particularly meticulous about that. We rinse the pulmonary artery and the venous anastomosis and deair antegradely through an open left atrial anastomosis, both from the pulmonary artery side and from the left atrial side. We make absolutely certain to achieve a good deairing on the first graft, because as the table is rotated with that graft down, an embolic event is more likely to occur. I would appreciate your comments on how you deair the allograft.

Dr. Bavaria. We have actually looked at this point in great detail. Of the five strokes, only one of them was an embolic air stroke (the patient recovered completely); three of them were caused by embolic particulate matter, and one of them was the result of a low-flow situation.

We deair in a standard fashion. I make a wide open left atrial hole before removing the left atrial clamp and actually allow quite a bit of blood to flow out. The pulmonary artery is left partially open, and the lungs are inflated a little bit before the left atrial clamp is released. Basically, it is the St. Louis method that Dr. Larry Kaiser brought with us. We also place the patient in the Trendelenburg position and try very hard to keep an intimato-intima anastomosis. The one change we have made is to now institute perioperative heparin therapy (2000 units intravenously before clamps are placed). 